\title{
A Coupled Structural-Magnetic Strain Model for Magnetostrictive Transducers
}

\author{
Marcelo J. Dapino* Ralph C. Smith ${ }^{\dagger}$ and Alison B. Flatau ${ }^{\ddagger}$ \\ * $\ddagger$ Aerospace Engineering \& Engineering Mechanics, Iowa State University, Ames, IA 50011 \\ $\dagger$ Center for Research in Scientific Computation, North Carolina State University, Raleigh, NC 27695
}

\begin{abstract}
The growing interest in giant magnetostrictive materials for generation of strains in smart structure systems motivates the development of increasingly accurate performance prediction and optimization tools. We propose a model for the strains generated by magnetostrictive materials in response to applied magnetic fields. The direct or magnetostrictive effect is modeled by considering active and passive components of the strain. The active or external component, associated with the alignment of magnetic moments with the external magnetic field, is modeled with a ferromagnetic hysteresis model in combination with a quartic magnetostriction law. The passive or internal component, associated with the elastic response of the transducer materials as they vibrate, is modeled through force balancing which yields a wave equation with magnetostrictive inputs. The effect of stress on the magnetization of the magnetostrictive core, or the magnetomechanical effect, is implemented by considering a 'law of approach' to the anhysteretic magnetization caused by stress. This provides a representation of the bi-directional coupling between the magnetic and elastic states. It is demonstrated that the model accurately characterizes the magnetic hysteresis and the strains output by a prototypical transducer.
\end{abstract}

Keywords: Magnetostriction, coupled magnetomechanical model, transducers, Terfenol-D

\section{Introduction}

This paper presents recent advances on the modeling of strains generated by transducer systems which utilize magnetostrictive materials as the active element. By virtue of the reciprocal nature of magnetostrictive materials, such transducer systems are capable of providing both actuation, characterized by magnetic-to-elastic energy transduction, and sensing, characterized by elastic-to-magnetic energy transduction. One crucial aspect of this two-way energy exchange is that neither transduction mechanism occurs independently, but rather they both occur simultaneously during transducer operation. In the direct or magnetostrictive effect (James Joule, 1842), the action of a magnetic field and ensuing magnetization induces strains in the magnetostrictive core. These strains are in turn associated with a stress field which affects the magnetic state, effect known as the inverse magnetostrictive effect or simply the magnetomechanical effect.

The close connection between magnetostriction and the magnetic behavior under stress has been long recognized, and extensive experimental evidence on the magnetomechanical effect has been documented in the literature $[1,2,3]$. In recent years there has been renewed interest on this phenomenon $[4,5]$ because of its relevance for technological applications such as non-destructive evaluation and sensing. Interest in the effects of stress on the performance of highly magnetostrictive materials, such as Terfenol-D, is also motivated by the need for increased levels of adaptability in smart structure systems.

In this paper, we extend the model derived in [6, 7], which includes magnetic hysteresis and nonlinearities as well as structural vibrations, to incorporate the magnetomechanical effect in a magnetostrictive rod as it drives a transducer. The model is illustrated in the context of the Terfenol-D transducer depicted in Figure 1, but is not limited to this application. The present approach may be implemented for other transducer materials such as nickel.

*(Correspondence)- Email: marcelod@iastate.edu; Telephone: 515-294-0084

†Email: rsmith@eos.ncsu.edu; Telephone: 919-515-7552

‡Email: abf@iastate.edu; Telephone: 703-306-1361 
Because the model quantifies the full magnetomechanical coupling exhibited by the material, it provides a more comprehensive representation of the physics of the system than does the uncoupled model. The model is derived under the assumption that the transducer is operated in quasistatic, temperature independent conditions and that the material is maintained in an approximately isotropic state.

The uncoupled formulation, modified to accommodate additional terms arising from the overall coupled approach, is summarized in Section 2. The magnetomechanical effect model employed is discussed in Section 3, while the complete, coupled model is compiled in Section 4. In Section 5, we compare model results with experimental data.

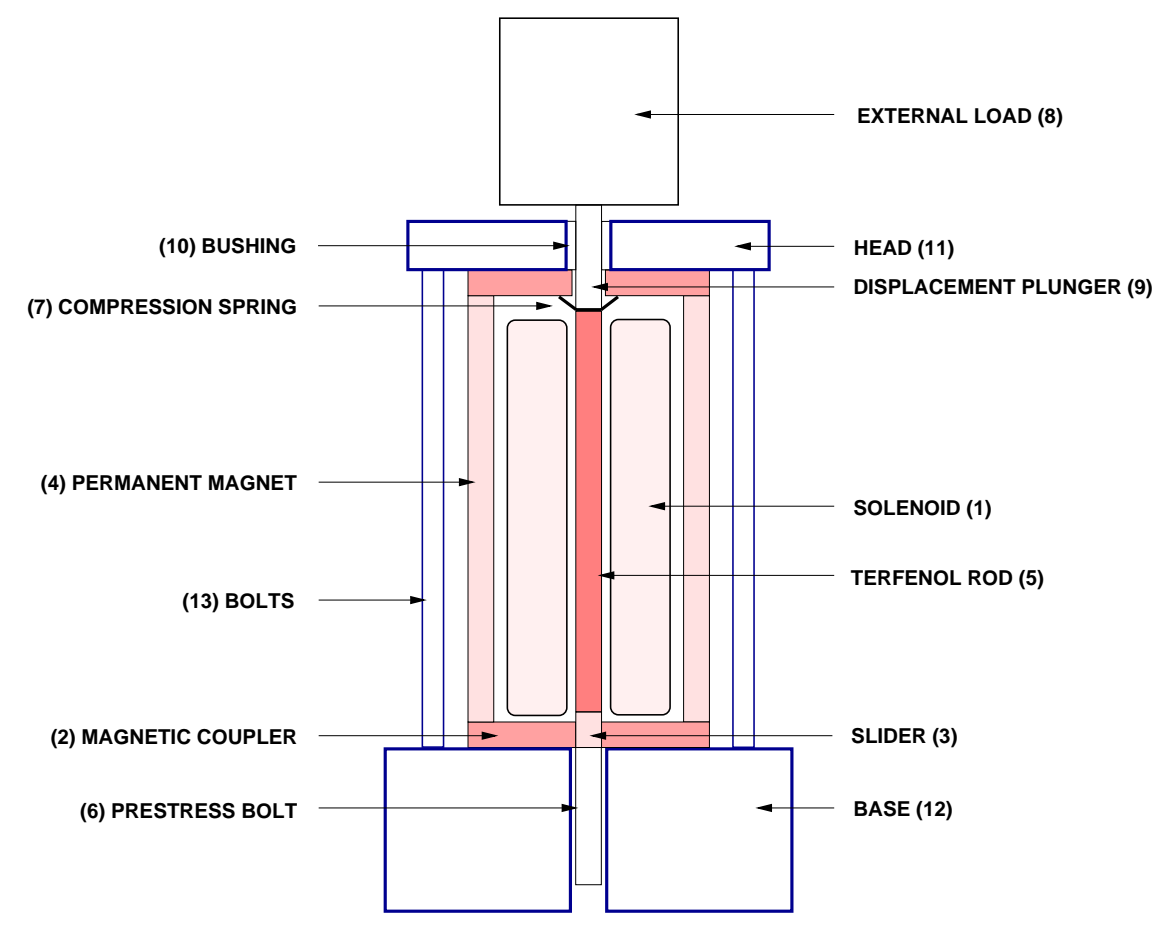

Figure 1: Cross section of the prototypical Terfenol-D transducer employed in the model.

\section{Magnetostrictive Effect}

In previous investigations, the magnetostrictive effect has been modeled in three stages [6, 7]. In the first stage, application of a magnetic field $H$ produces a bulk magnetization $M$. The hysteresis and saturation effects present in the $M-H$ relationship, illustrated in Figure 2(a), are modeled through a mean field model of ferromagnetic hysteresis originally developed by Jiles and Atherton $[8,9,10]$. The second stage involves the characterization of the magnetostriction due to the alignment of magnetic moments with the applied magnetic field and ensuing magnetization. We call this magnetostriction $\lambda$, to distinguish it from the total magnetoelastic strain $\varepsilon$. The most salient characteristics of the $\lambda-M$ relation are depicted in Figure 2(b). The model employed at this stage is a truncated empirical series expansion symmetric about $M=0$. The third aspect that needs to be characterized is the total strain $\varepsilon$, which incorporates both magnetic effects and the elastic properties of the transducer. Typical issues to address in the $\varepsilon-H$ relationship are hysteresis, nonlinearities, and saturation effects, as indicated in Figure 2(c). Modeling of the structural vibrations of the magnetostrictive core is posed through force balancing, in the form of a PDE equation which includes the intrinsic magnetostriction, system compliance, internal damping, and corresponding boundary conditions associated with the mechanical transducer design.

It is noted that the rate of change of magnetization with time can be expressed as

$$
\frac{d M}{d t}=\left(\frac{\partial M}{\partial H}\right) \frac{d H}{d t}+\left(\frac{\partial M}{\partial \sigma}\right) \frac{d \sigma}{d t}
$$

which motivates treating the field and stress components independently. The remainder of this section deals with the field-dependent magnetization and corresponding derivative $\frac{\partial M}{\partial H}$. 
STAGE 1

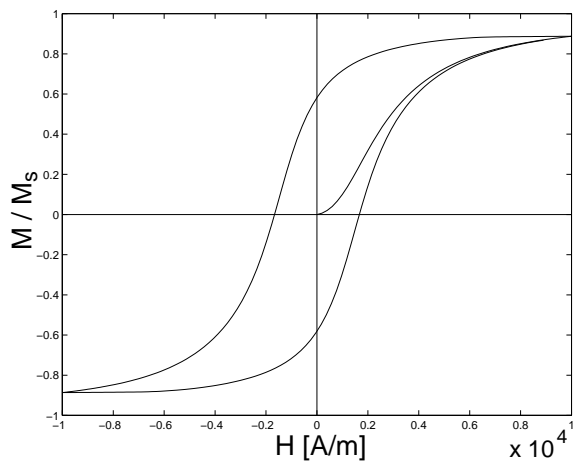

(a)
STAGE 2

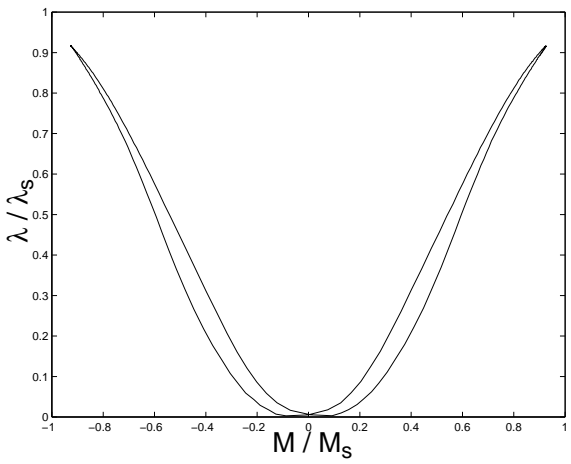

(b)
STAGE 3

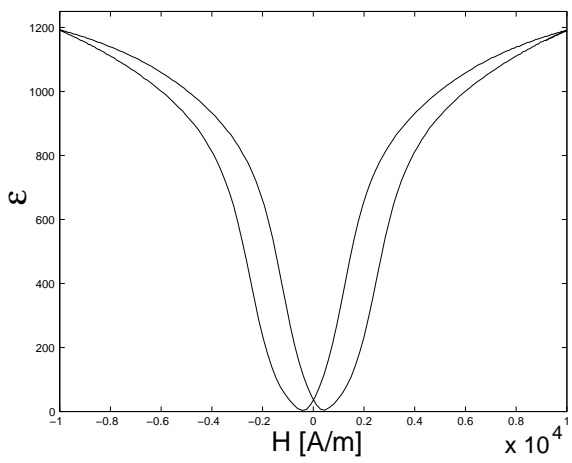

(c)

Figure 2: Model simulations depicting stages 1-3 of the magnetostrictive effect: (a) constant-stress, relative magnetization $\frac{M}{M_{s}}$ versus applied field $H$, with $M_{s}$ the saturation magnetization, (b) relative magnetostriction $\frac{\lambda}{\lambda_{s}}$ versus relative magnetization, with $\lambda_{s}$ the saturation magnetostriction, and (c) total strain $\varepsilon$ versus applied field $H$.

\section{$2.1 \quad$ Field-Dependent Magnetization}

As discussed in [11, 12], the magnetization process taking place in ferromagnetic materials in response to an applied magnetic field can be explained by considering two related mechanisms. The first mechanism is that domain walls (the transition layers between highly aligned regions termed magnetic domains) move under the influence of the magnetic field in such a way that favorably oriented domains grow at the expense of unfavorably oriented domains. The wall thickness is determined by a fine balance between the anisotropy, which tends to make the walls thinner, and the Weiss-type interaction coupling between atomic magnetic moments, which tends to make the walls thicker. The second mechanism involves the rotation of magnetic moments within domains towards the field direction.

As detailed in [7], the minimization of an appropriate thermodynamic potential $A$ with respect to the magnetization yields the expression

$$
H_{e}=\frac{1}{\mu_{0}} \frac{\partial A}{\partial M}=H+\alpha M+\frac{3}{2 \mu_{0}}\left[\sigma \frac{\partial \lambda}{\partial M}+\lambda \frac{\partial \sigma}{\partial M}\right]
$$

for the effective magnetic field, where $\mu_{0}$ is the permeability of free space. Note that $H_{e}$ is composed of: (i) the applied magnetic field $H$, (ii) an interaction field $\alpha M$ responsible for the alignment of neighboring magnetic moments, and (iii) a component due to magnetoelastic interactions, $H_{\sigma}=\frac{3}{2 \mu_{0}}\left[\sigma \frac{\partial \lambda}{\partial M}+\lambda \frac{\partial \sigma}{\partial M}\right]$.

Maxwell-Boltzmann statistics can be used to quantify the anhysteretic magnetization through the Langevin equation

$$
M_{a n}=M_{s}\left[\operatorname{coth}\left(\frac{H_{e}}{a}\right)-\frac{a}{H_{e}}\right]
$$

Here, $M_{s}$ represents the saturation magnetization while the constant $a$, representing the effective domain density, is treated as a parameter to be identified. Although the Langevin equation works well for paramagnetic materials, it does not provide a good description of the magnetization phenomenon in ferromagnetic materials, in which the motion of domain walls is impeded by imperfections. These imperfections or pinning sites, usually attributed to crystal defects and the presence of inclusions such as dysprosium in Terfenol-D, form energy wells which are energetically favorable for domain wall attachment.

The effects of pinning sites on domain wall motion under constant stress conditions have been assessed in [8, 9, 10], through consideration of reversible $M_{r e v}$ and irreversible $M_{i r r}$ components of the magnetization. For low magnetic field intensities about an equilibrium level, the domain walls bend reversibly while remaining attached to pinning sites. As the applied magnetic field is increased, the domain walls achieve sufficient energy to break free from pinning sites while moving up the energy well where they were originally located, and attach to remote sites where the energy configuration is favorable. The effect of the energy losses caused by pinning of domain walls manifests itself as hysteresis in the magnetization process. 
Energy balancing yields an expression for the rate of change of irreversible magnetization with applied field [13],

$$
\frac{\partial M_{i r r}}{\partial H}=\frac{M_{a n}-M_{i r r}}{\frac{\delta k}{1-c}}\left(\frac{\partial H_{e}}{\partial H}\right)
$$

where the constant $k$ quantifies the energy needed to break pinning sites, $c$ is a reversibility coefficient which quantifies the flexibility of the domain walls, and $\delta$ has the value +1 when $\frac{\partial H}{\partial t}>0$ and -1 when $\frac{\partial H}{\partial t}<0$. The parameter $\delta$ ensures that the energy lost to pinning always opposes changes in the magnetization. Differentiation of (1) with respect to $H$, along with application of the chain rule yields

$$
\frac{\partial H_{e}}{\partial H}=1+\left[\alpha+\frac{3}{2 \mu_{0}} \sigma \frac{\partial}{M}\left(\frac{\partial \lambda}{\partial M}\right)+\frac{3}{\mu_{0}} \frac{\partial \sigma}{\partial M} \frac{\partial \lambda}{\partial M}+\frac{3}{2 \mu_{0}} \lambda \frac{\partial}{\partial M}\left(\frac{\partial \sigma}{\partial M}\right)\right] \frac{\partial M}{\partial H}
$$

To facilitate model implementation, the magnetic coupling and magnetoelastic interactions are combined in a single unitless term which needs to be identified upon characterization of $\lambda-M$,

$$
\widetilde{\alpha}(M, \sigma)=\alpha+\frac{3}{2 \mu_{0}} \sigma \frac{\partial}{M}\left(\frac{\partial \lambda}{\partial M}\right)+\frac{3}{\mu_{0}} \frac{\partial \sigma}{\partial M} \frac{\partial \lambda}{\partial M}+\frac{3}{2 \mu_{0}} \lambda \frac{\partial}{\partial M}\left(\frac{\partial \sigma}{\partial M}\right) .
$$

The substitution of (5) into (4), and (4) into (3), application of the chain rule, and recognition from [13] that $\frac{\partial M}{\partial M_{i r r}} \approx 1$, yields the relation

$$
\frac{\partial M_{i r r}}{\partial H}=\frac{M_{a n}-M_{i r r}}{\frac{\delta k}{1-c}-\widetilde{\alpha}(M, \sigma)\left(M_{a n}-M_{i r r}\right)}
$$

which characterizes the irreversible component of the field-dependent magnetization.

Following ideas presented in [10], the reversible component of the field-dependent magnetization is taken to be proportional to the displacement from the anhysteretic magnetization, which may be expressed in differential form as follows

$$
\frac{\partial M_{r e v}}{\partial H}=c\left(\frac{M_{a n}}{\partial H}-\frac{\partial M_{i r r}}{\partial H}\right)
$$

where the coefficient $c$ can be estimated from the ratio of the initial and anhysteretic susceptibilities [14] or through fit to experimental data.

Finally, the reversible and irreversible terms, (7) and (6), are added to give

$$
\frac{\partial M}{\partial H}=(1-c) \cdot \frac{M_{a n}-M_{i r r}}{\frac{\delta k}{1-c}-\widetilde{\alpha}(M, \sigma)\left(M_{a n}-M_{i r r}\right)}+c \cdot \frac{M_{a n}}{\partial H}
$$

which characterizes the total magnetization arising from the application of a magnetic field.

\subsection{Magnetostriction Model}

Since the magnetostriction is structure sensitive, assessing a general $\lambda-M$ relationship is not practical in the context of the present transducer model, despite the fact that the saturation magnetostriction $\lambda_{s}$ can be explicitly identified from $\lambda_{111}$ and $\lambda_{100}$ (Lee [15] provides an extensive review of the magnetostriction of anisotropic materials.) However, there is one simple case in which the relationship $\lambda-M$ can be identified, namely when the magnetic moments are forced to align perpendicular to the direction of applied field. This is accomplished in the case of polycrystalline materials such as Terfenol-D by proper crystal growth during the manufacturing process and by applying sufficiently large compressive preloading during operation. In this case the dominant magnetization process is domain rotation, and energy minimization yields the single-valued quadratic law, $\lambda(M)=\frac{3}{2} \frac{\lambda_{s}}{M_{s}^{2}} M^{2}$.

An alternative but not totally unrelated approach consists of formulating an empirical model based on a series expansion symmetric about $M=0$,

$$
\lambda(M)=\sum_{i=0}^{\infty} \gamma_{i} M^{2 i}
$$


where the coefficients $\gamma_{i}$ are determined from experimental data [5]. The series is often truncated after $i=1$, yielding a model equivalent to the quadratic law when $\gamma_{1}=\frac{3}{2} \frac{\lambda_{s}}{M_{s}^{2}}$, or after $i=2$, yielding a quartic model. The parameters $\gamma_{1}$ and $\gamma_{2}$ of the quartic model are identified by solving simultaneously the constraints at magnetic saturation $M_{s}$ and at the inflection point $M_{0}$,

$$
\begin{aligned}
\lambda\left(M_{s}\right) & \equiv \gamma_{1} M_{s}^{2}+\gamma_{2} M_{s}^{4}=\lambda_{s} \\
\text { (b) } \frac{\partial^{2} \lambda}{\partial M^{2}}\left(M_{0}\right) & \equiv 2 \gamma_{1}+12 \gamma_{2} M_{0}^{2}=0
\end{aligned}
$$

A more sophisticated model capable of including hysteresis effects is that formulated by Sablik and Jiles [16]. However, for the remainder of this discussion the quartic magnetostriction model is employed. The constant term $(i=0)$ will be ignored since it corresponds to the elastic strains considered in Section 2.3.

\subsection{Transducer Structural Dynamics}

The magnetostriction $\lambda$ given by (9) quantifies the reorientation of magnetic moments towards the direction of the applied field. As detailed in [7], this magnetostriction is analogous to the term $d_{33} H$ in linear models but is inherently nonlinear and hysteretic through both the magnetization $M$ and $\lambda-M$ itself. It ignores, however, the elastic properties of the magnetostrictive material as it vibrates, represented in the linear models by $s^{H} \sigma$. In this section, a PDE system is formulated which models the elastic response of the magnetostrictive material and other transducer components. The input to this PDE is formulated through the magnetostriction $\lambda$, which constitutes an 'internal force' commanding the vibrations of the transducer. The solution to the PDE is the longitudinal displacements $u(t, x)$ relative to the prestressed position. Additional details regarding this PDE formulation are provided in [7].

The structural dynamics are modeled through consideration of the magnetostrictive rod (5), prestress bolt (6), spring (7), and mass load (8) for the transducer depicted in Figure 1. The prestress bolt provides a stress $\sigma_{0}<0$ by compressing the magnetostrictive core against the spring, modeled by a linear spring $k_{L}$ and dashpot $c_{L}$. The rod is assumed to have length $L$, cross sectional area $A$, and longitudinal coordinate $x$. The material density is $\rho$, the elastic modulus is $E$, and the internal damping is $c_{D} .{ }^{1}$ Finally, the external load is modeled by a point mass $m_{L}$.

The total stress in the rod is given by the expression

$$
\sigma(t, x)=E \frac{\partial u}{\partial x}(t, x)+c_{D} \frac{\partial^{2} u}{\partial x \partial t}(t, x)-E \lambda(t, x)+\sigma_{0}
$$

where the terms on the right hand side represent respectively the linear elasticity at small displacements, KelvinVoigt damping, magnetostriction-derived stress, and prestress. Force balancing then yields the dynamic model for the longitudinal displacements and strains.

For implementation purposes, the model is formulated in weak or variational form by multiplying the strong form by test functions $\phi$ followed by integration throughout the length of the rod. This reduces the smoothness requirements on the finite element basis since displacements and test functions need be differentiated only once compared to the second derivatives present in the strong form. The space of test functions is $V=H_{L}^{1}(0, L) \equiv\{\phi \in$ $\left.H^{1}(0, L) \mid \phi(0)=0\right\}$, so that for all $\phi(x) \in V$,

$$
\begin{aligned}
\int_{0}^{L} \rho A \frac{\partial^{2} u}{\partial t^{2}}(t, x) \phi(x) d x= & -\int_{0}^{L}\left[E A \frac{\partial u}{\partial x}(t, x)+c_{D} A \frac{\partial^{2} u}{\partial x \partial t}(t, x)-E A \lambda(t, x)\right] \frac{\partial \phi}{\partial x}(x) d x \\
& -\left[k_{L} u(t, L)+c_{L} \frac{\partial u}{\partial t}(t, L)+m_{L} \frac{\partial^{2} u}{\partial t^{2}}(t, L)\right] \phi(L) .
\end{aligned}
$$

The solution $u(t, x)$ to (11) defines the longitudinal displacements about the prestressed position. Once the displacements are computed, the strains are evaluated by taking derivatives with respect to position, $\varepsilon(t, x)=\frac{\partial u}{\partial x}(t, x)$, and the material stresses $\sigma(t, x)$ are calculated directly from (10). Note that the stress at the rod end $\sigma(t, L)$ may be equivalently calculated from the boundary condition $\sigma(t, L)=\frac{1}{A}\left[-k_{L} u(t, L)-c_{L} \frac{\partial u}{\partial t}(t, L)-m_{L} \frac{\partial^{2} u}{\partial t^{2}}(t, L)\right]$.

\footnotetext{
${ }^{1}$ The parameter $E$ lies between the elastic modulus at constant $H, E^{H}$, and at constant $B, E^{B}$. Since $E^{H}$ and $E^{B}$ depend upon the field intensity [17], so does $E$. However, for simplicity it is assumed that $E$ represents a nominal or operational material stiffness.
} 


\section{Magnetomechanical Effect}

In this section, we consider the stress-dependent magnetization through its corresponding derivative $\frac{\partial M}{\partial \sigma}$. A unifying description of the changes in magnetization due to the action of stress has been developed recently in $[4,5,18]$. In the model theory developed in [5] and implemented here, the main mechanism responsible for the magnetomechanical effect is the unpinning of domain walls that is caused by the application of stress. This unpinning allows the walls to move creating a corresponding magnetization change. This magnetization change is such that the total magnetization approaches the anhysteretic condition, in which the domain configuration has achieved a state of energy minimization. This effect is depicted in the schematic $M-H$ loop of Figure 3(a), where the magnetization in the ferromagnetic sample is measured for varying compressive stresses (while leaving the magnetic field constant) starting at locations above and below the anhysteretic, points A and B respectively.

A second, related effect is the change in the anhysteretic curve itself under the action of stress. Application of tensile stress produces an increase in both the slope of the $M-H$ loop and the remanent magnetization value, while compressive stress produces a shearing of the $M-H$ loop. This effect is illustrated in Figure 4, where the influence of stress on both the anhysteretic and total magnetization is considered for four stress levels: $-10,0,+10$, and $+20 \mathrm{MPa}$.

The net result of both effects considered in combination is that a monotonically increasing compressive stress causes the value of $\mathrm{X}$ to be approached (Figure 3(a)), while the value of $\mathrm{X}$ itself decreases (Figure 4). The magnetomechanical effect model presented in this section is then formulated in the context of: (i) the effect of stress on the anhysteretic magnetization and (ii) a law of approach to the anhysteretic magnetization upon application of stress.

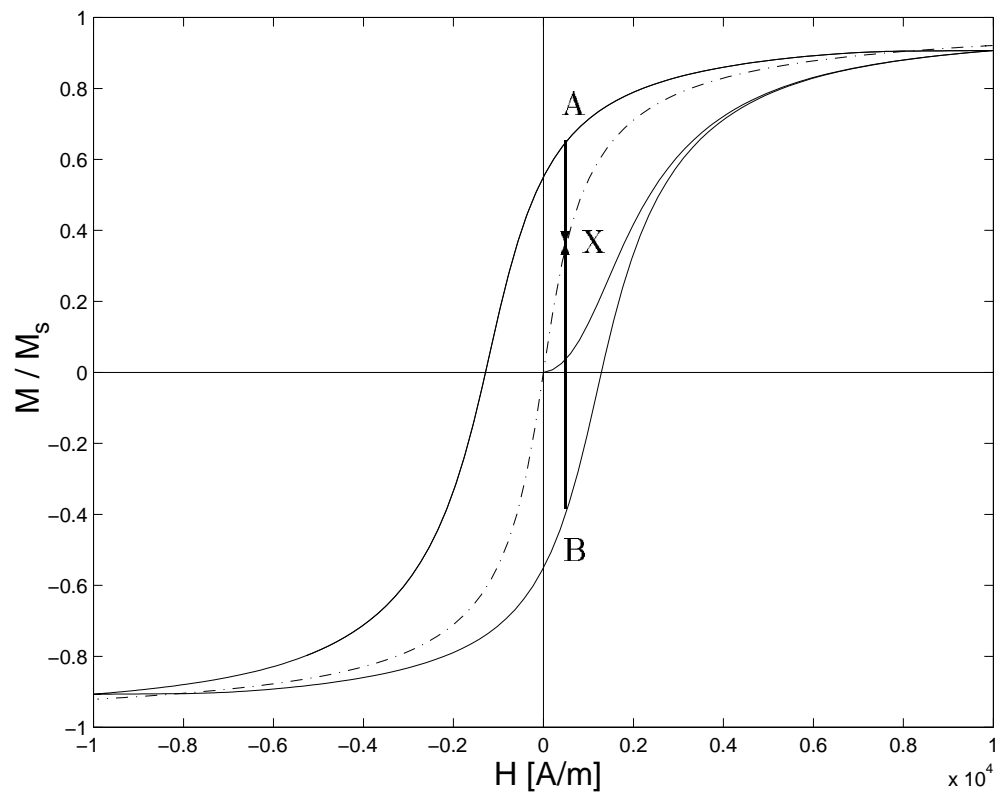

(a)
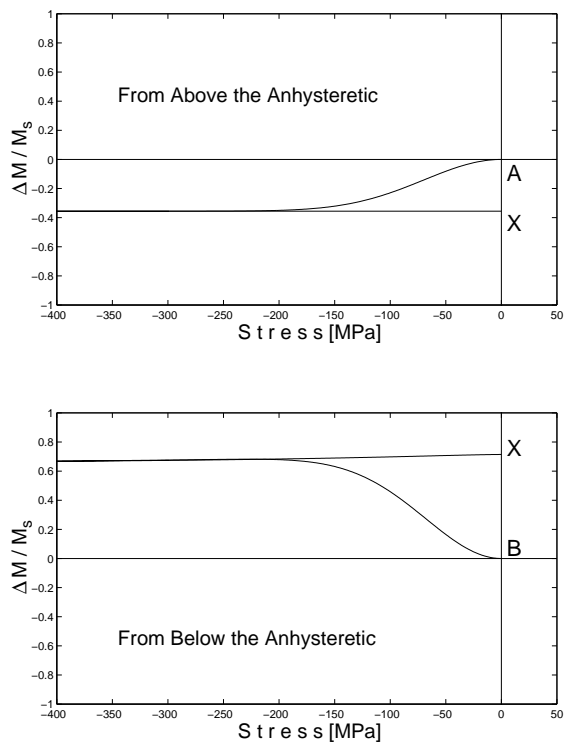

(b)

Figure 3: Schematic representation of the approach to the anhysteretic magnetization under stress (after Pitman [4]). (a) Characteristic $M-H$ loop showing the approach from positions above and below the anhysteretic (A and B, respectively) for fixed $H$. In either case, $M$ moves towards point $\mathrm{X}$ on the anhysteretic curve. (b) Detail of the variation in magnetization $\Delta M / M_{s}$ under application of stress starting at point $\mathrm{A}$ above the anhysteretic magnetization $(\mathrm{A} \rightarrow \mathrm{X})$, and at point $\mathrm{B}$ below the anhysteretic magnetization $(\mathrm{B} \rightarrow \mathrm{X})$.

\subsection{Anhysteretic Magnetization}

The anhysteretic magnetization calculated in (2) should be interpreted as a function of both $H$ and the particular value of the prevailing magnetization $M$, with both operating through the effective field $H_{e}$ given by (1). For instance, at point $\mathrm{A}$ in Figure 5, the material is in a state of equilibrium in the presence of zero applied field, due to 

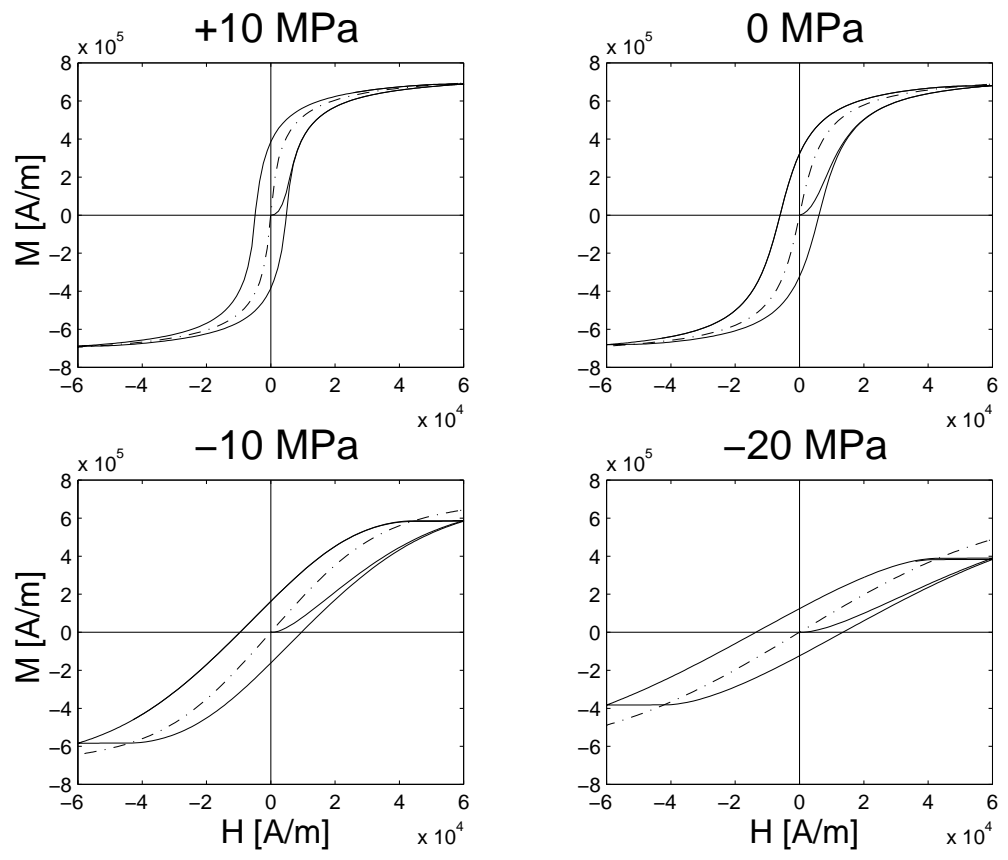

Figure 4: Model simulations representing the effect of stress on the total magnetization ( $\longrightarrow$ ), and on the global anhysteretic magnetization $(-\cdot-)$, for $+10,0,-10$, and $-20 \mathrm{MPa}$.

the coupling between magnetic moments $\alpha M$ and the magnetoelastic interactions $H_{\sigma}$ in the material. Application of a small magnetic field perturbs this equilibrium slightly, giving a new value of $M_{a n}$. Applying small field increments successively, the two-valued local anhysteretic curve shown in Figure 5 is obtained. Note, however, that global equilibrium is never achieved in this case, because coupling and the presence of pinning sites do not allow sufficient relaxation.

In contrast, the 'global' anhysteretic magnetization represents the locus of points in which, for a given field level $H$ and elastic state $\sigma$, the material reaches an equilibrium associated with the minimization of the total energy. The global anhysteretic, $\bar{M}_{a n}$, is a single-valued function of $H$ as illustrated in Figure 5, and is the foundation of the magnetomechanical effect model implemented here. It is noted that in the absence of magnetic coupling $(\alpha=0)$, the local and global anhysteretic curves coincide.

The global anhysteretic depends significantly on the stress state in the material. This dependency is posed in terms of the effective field $H_{e}$, by recognizing from [5] that the global anhysteretic magnetization under a field $H$ and stress $\sigma$ is equivalent to the global anhysteretic magnetization under field $H_{e}$ and zero stress, $\bar{M}_{a n}(H, \sigma)=\bar{M}_{a n}\left(H_{e}, 0\right)$. Assuming the quartic magnetostriction model and substituting into (1), yields the effective field

$$
H_{e}=H+\alpha M+\frac{3}{2 \mu_{0}}\left[\left(2 \gamma_{1} M+4 \gamma_{2} M^{3}\right) \sigma+\left(\gamma_{1} M^{2}+\gamma_{2} M^{4}\right) \frac{\partial \sigma}{\partial M}\right]
$$

Upon definition of a new effective coupling term

$$
\bar{\alpha}(M, \sigma)=\alpha+\frac{3}{2 \mu_{0}}\left[\left(2 \gamma_{1}+4 \gamma_{2} M^{2}\right) \sigma+\left(\gamma_{1}+\gamma_{2} M^{2}\right) M \frac{\partial \sigma}{\partial M}\right],
$$

the effective field may be written in the form

$$
H_{e}=H+\bar{\alpha}(M, \sigma) M .
$$

The global anhysteretic is then the locus of points which satisfy identically the modified Langevin equation (2),

$$
\bar{M}_{a n}=M_{s}\left[\operatorname{coth}\left(\frac{H+\bar{\alpha}\left(\bar{M}_{a n}, \sigma\right) \bar{M}_{a n}}{a}\right)-\left(\frac{a}{H+\bar{\alpha}\left(\bar{M}_{a n}, \sigma\right) \bar{M}_{a n}}\right)\right] .
$$




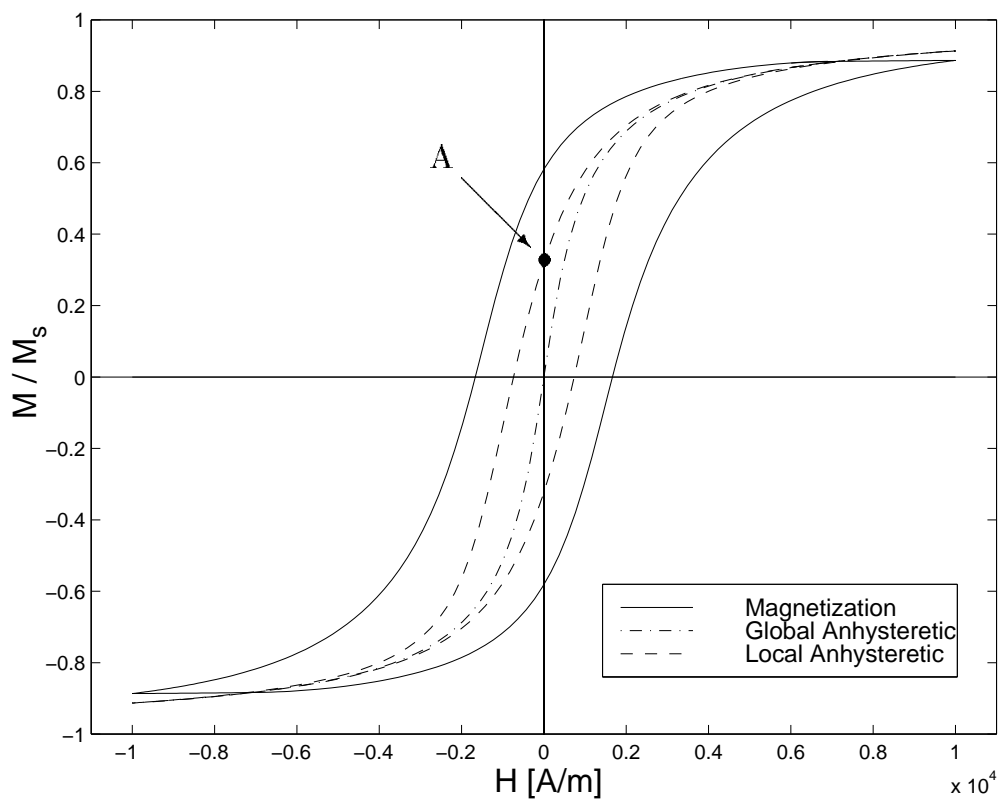

Figure 5: Model simulations showing the relative magnetization $M / M_{s}$, relative local anhysteretic $M_{a n}(H, M, \sigma) / M_{s}=$ $\operatorname{coth}[(H+\widetilde{\alpha}(M, \sigma) M) / a]-[a /(H+\widetilde{\alpha}(M, \sigma) M)]$, and relative global anhysteretic $\bar{M}_{a n}(H, \sigma) / M_{s}=\operatorname{coth}[(H+$ $\left.\left.\bar{\alpha}\left(\bar{M}_{a n}, \sigma\right) \bar{M}_{a n}\right) / a\right]-\left[a /\left(H+\bar{\alpha}\left(\bar{M}_{a n}, \sigma\right) \bar{M}_{a n}\right)\right]$, as a function of $H$.

\subsection{Stress-Dependent Magnetization}

The behavior of the magnetostrictive core depends on more than just the externally applied stress $\sigma$ and magnetic field $H$. In fact, the magnetic history of the specimen, or equivalently the displacement from the anhysteretic condition in the case of major $M-H$ loops, is what determines the direction of changes in magnetization. According to the experimental observations documented in $[3,8,19]$, the direction in which the magnetization changes with applied stress is independent of the sign of the stress, for small stresses and when the magnetization is sufficiently distant from the anhysteretic. It is then inferred that the direction of change is dependent not on the stress itself, but on a quantity which is independent of the sign of the stress. In this context, Jiles hypothesized in [5] that this quantity is the elastic energy per unit volume, $W=\sigma^{2} /(2 E)$, which is clearly independent of the sign of $\sigma$ and which may be responsible for some unpinning of domain walls. The 'law of approach' to the anhysteretic condition is then formulated as follows: the rate of change of magnetization with elastic energy is proportional to the displacement of the prevailing magnetization from the anhysteretic magnetization. As before (Section 2.1), this may be posed via irreversible and reversible components. The law of approach as applied to the irreversible component then gives

$$
\frac{\partial M_{i r r}}{\partial W}=\frac{1}{\xi}\left(\bar{M}_{a n}-M_{i r r}\right),
$$

where $\xi$ is a coefficient to be identified with dimensions of energy per unit volume. Making the substitution $\frac{\partial W}{\partial \sigma}=\frac{\sigma}{E}$ in (12), along with application of the chain rule, permits writing the rate of change of $M_{i r r}$ with $\sigma$ as follows,

$$
\frac{\partial M_{i r r}}{\partial \sigma}=\frac{\sigma}{E \xi}\left(\bar{M}_{a n}-M_{i r r}\right) .
$$

A procedure similar to that employed in the field-dependent case yields the rate of change of reversible magnetization with stress,

$$
\frac{\partial M_{r e v}}{\partial \sigma}=c\left(\frac{\partial \bar{M}_{a n}}{\partial \sigma}-\frac{\partial M_{i r r}}{\partial \sigma}\right),
$$

in which $c$ is the coefficient defined in $(7)$. 
The reversible and irreversible terms, (14) and (13), are added, to give

$$
\frac{\partial M}{\partial \sigma}=(1-c) \cdot \frac{\sigma}{E \xi}\left(\bar{M}_{a n}-M_{i r r}\right)+c \cdot \frac{\partial \bar{M}_{a n}}{\partial \sigma}
$$

which characterizes the magnetization arising from the application of stress.

\section{Coupled Model}

In the presence of both an applied magnetic field $H$ and stress $\sigma$, the total magnetization is dictated by the superposition of the field- and stress-dependent components given in (8) and (15). For implementation purposes, however, we consider instead the governing differential equation

$$
\frac{d M_{i r r}}{d t}=\left(\frac{\partial M_{i r r}}{\partial H}\right) \cdot \frac{d H}{d t}+\left(\frac{\partial M_{i r r}}{\partial \sigma}\right) \cdot \frac{d \sigma}{d t}
$$

for the total irreversible magnetization $M_{i r r}$ which arises from the superposition of field and stress.

It is noted that the relationship between applied current $I(t)$ and magnetic field $H(t, x)$ generated in a solenoid is often characterized by $H(t)=n I(t)$, where $n=\frac{\text { No.turns }}{\text { length }}$. Experimental evidence on research transducers indicates that $H=n I$ does not provide a sufficiently accurate characterization of the magnetic field, so an empirical positiondependent filter $\Phi(x)$ is considered, which accounts for end effects, demagnetizing factors, and overall losses in the magnetic circuit. Thus, the time and spatial dependencies of the field are formulated via $H(t, x)=I(t) \Phi(x) .{ }^{2}$ Applying the chain rule and substituting (6) and (12) into (16) yields

$$
\begin{aligned}
\frac{\partial M_{i r r}}{\partial t}(t, x)= & {\left[\frac{M_{a n}(t, x)-M_{i r r}(t, x)}{\frac{\delta k}{1-c}-\widetilde{\alpha}(M, \sigma)\left(M_{a n}(t, x)-M_{i r r}(t, x)\right)}\right] \cdot \frac{\partial I}{\partial t}(t) \cdot \Phi(x) } \\
& +\frac{\sigma(t, x)}{E \xi} \cdot\left(\bar{M}_{a n}(t, x)-M_{i r r}(t, x)\right) \cdot \frac{\partial \sigma}{\partial t}(t, x),
\end{aligned}
$$

which after integration gives the irreversible component of the magnetization, $M_{i r r}(t, x)$.

The reversible magnetization is calculated directly upon integration and subsequent superposition of (7) and (13),

$$
M_{r e v}(t, x)=c\left[M_{a n}(t, x)+\bar{M}_{a n}(t, x)\right]-c M_{i r r}(t, x) .
$$

The total magnetization arising from application of both a field and stress is then

$$
M(t, x)=c\left[M_{a n}(t, x)+\bar{M}_{a n}(t, x)\right]+(1-c) M_{i r r}(t, x),
$$

which incorporates the reversible and irreversible components of magnetization. It should be noted that in the case of constant stress $\left(\frac{\partial \sigma}{\partial t}=0\right)$ or constant field $\left(\frac{\partial I}{\partial t}=0\right)$, the expression reduces to the individual components characterized in (8) and (15).

\section{$5 \quad$ Model Validation}

\section{$5.1 \quad$ Experimental Setup}

In this section, the model presented in Section 4 is applied to a transducer developed at Iowa State University (the configuration of which follows the general guidelines of Figure 1), and used to characterize the time-dependent magnetization and displacement produced by the transducer in response to an applied current $I(t)$. These calculations are compared to experimental measurements collected in this transducer. The driver was a 4.53 in (115 mm) long, 0.5 in $(12.7 \mathrm{~mm})$ diameter, monolithic $\mathrm{Tb}_{0.3} \mathrm{Dy}_{0.7} \mathrm{Fe}_{1.9}$ rod manufactured using the Bridgman process. Surrounding

\footnotetext{
${ }^{2}$ Note: (i) $\Phi(x)$ is equal to $n$ in the idealized situation of an infinitely long, lossless solenoid, and (ii) an alternative approach for identification of $H-I$ consists of solving the Àmpère's law or the Biot Savart law. Several software packages utilize finite element analysis to accomplish this.
} 
the rod were an innermost single-layer, 150-turn pickup coil, used for magnetic induction measurements, and a sixlayer, 900-turn drive coil connected to a Techron 7780 current control amplifier. Both solenoids were wound in-house with 26 AWG magnet wire.

Steel end caps and a cylindrical Alnico V permanent magnet provided flux closure. For the experiments reported here, the permanent magnet was demagnetized to obtain unbiased operation. The displacement plunger was guided by a nylon bushing mounted within an aluminum head, which was held in place by non-magnetic steel bolts attached to a heavy stainless steel base. The $0.7 \mathrm{~Hz}$ sinusoidal reference signal input to the amplifier was provided by a Tektronix 2642A Fourier Personal Analyzer, which was also used to record the pick-up coil, drive current, drive voltage and displacement signals. The external load was a $0.5 \mathrm{~kg}$ mass threaded onto the displacement plunger.

The compressive preloading necessary to avoid tensile stresses on the moderately brittle Terfenol-D rod was provided by a steel bolt located within the transducer base. The bolt pushed the rod against a Belleville washer, whose force-displacement characteristic curve was measured in an MTI Phoenix compression machine. During preloading, the bolt was tightened until the plunger's displacement matched the displacement in the washer's characteristic curve corresponding to the desired force level.

The displacement at the rod tip was measured with a Lucas Schaevitz LVM-110 linear variable differential transducer equipped with a 050 -MHR probe, capable of $99.9923 \%$ linearity over \pm 0.050 in. The corresponding bulk strain, used for comparison to model simulations, was computed by dividing this displacement by $L$.

For identification of the $\Phi(x)$ functional, a series of flux measurements was conducted with a Hall effect probe (F. W. Bell 9500 series), located within the transducer to allow characterization of end effects, demagnetizing factors, and magnetic circuit nonlinearities. The amplitude of the flux measured adjacent to the surface of the rod at locations $3 \mathrm{~mm}$ apart over a longitudinal line, $\left|B_{\text {sur }}(t, x)\right|$, is equal to the magnetic field amplitude, $\left|H_{\text {sur }}(t, x)\right|$, because in air $B=H$ in the CGS units system. The filter was identified by taking the ratio $\frac{\left|H_{\text {sur }}(t, x)\right|}{|I(t)|}$ at several current levels, and a look-up table was used at the desired current level.

The magnetic induction was calculated by integration of the pickup coil signal $V_{p u}$, following the Faraday-Lenz law of magnetic induction, $B(t)=-\frac{1}{N_{p u} A_{p u}} \int_{0}^{t} V_{p u}(\tau) d \tau$. The parameter $A_{p u}$ is the mean cross sectional area of the pickup coil, and $N_{p u}$ was equal to 150 turns as indicated before. The magnetization used for comparison against model simulations was then computed from the magnetic constitutive equation $M=\frac{1}{\mu_{0}} B-H$.

\subsection{Parameter Estimation}

A total of fourteen parameters completely describe the coupled magnetic-structural model, of which six are magnetization parameters $\left(a, k, c, \alpha, M_{s}\right.$ and $\left.\xi\right)$, two are magnetostriction parameters $\left(M_{0}\right.$ and $\lambda_{s}$, or $\gamma_{1}$ and $\left.\gamma_{2}\right)$, three are material properties of the transducer driver $\left(E, \rho\right.$ and $\left.c_{D}\right)$, and three describe the load characteristics $\left(k_{L}, c_{L}\right.$ and $\left.m_{L}\right)$. The parameters were quantified in three manners: i) by direct measurement $\left(k_{L}, m_{L}\right.$ and $\rho$ ), ii) from published specifications for Terfenol-D $\left(M_{s}, E\right.$ and $\left.\lambda_{s}\right)$, and iii) by optimization through a least square fit to experimental data $\left(a, k, c, \alpha, M_{0}, c_{D}, c_{L}\right.$ and $\left.\xi\right)$. The optimization was performed in two steps. In the first, the five magnetization parameters and damping components were estimated from magnetization data. The second step consists of identifying the magnetostriction coefficients from strain data.

The magnetization parameters have been previously identified for ferromagnetic materials in general in [14], and for Terfenol-D in particular in the uncoupled case, in [6,20]. The parameter optimization performed for the coupled case reveals some differences in these four parameters compared to the previous work, which is most likely attributable to the effects of coupling.

For Terfenol-D, the nominal saturation magnetostriction is $\lambda_{s}=1000 \times 10^{-6}$. The saturation magnetization has been found to deviate little from the nominal $M_{s}=0.765 \times 10^{6} \mathrm{~A} / \mathrm{m}$. The optimized model parameters employed in the simulations are summarized in Table 1.

\subsection{Magnetization and Strain Results}

The mean-field model discussed in Section 2.1 provides a characterization of the magnetization $M$ in the transducer core in response to an applied field $H$. The performance of the model is demonstrated in Figure 6(a). It is noted that the model accurately characterizes the fundamental aspects of the magnetization characteristic curve. However, the model does not quantify the constricted behavior at low field levels, which is perhaps due to domain rotation effects. It is observed that the magnetization model does not include the moderate anisotropy typical of Terfenol-D.

The strain simulations are gauged against experimental data in Figure 6(b). The model accurately captures the nonlinearity, hysteresis, and saturation features present in the data. We note that the errors observed in the $M-H$ 
model propagate to the strain measurements through the magnetostriction law, a fact which explains some of the discrepancies present in the performance of the complete $\varepsilon-H$ model. Moreover, the approximate nature of the quartic law itself is thought to constitute an additional source for errors.

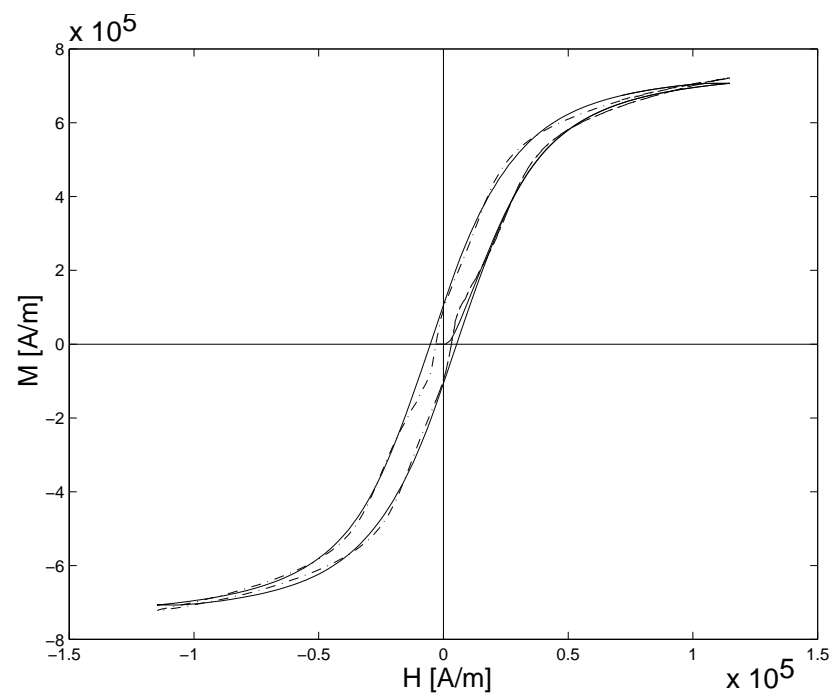

(a)

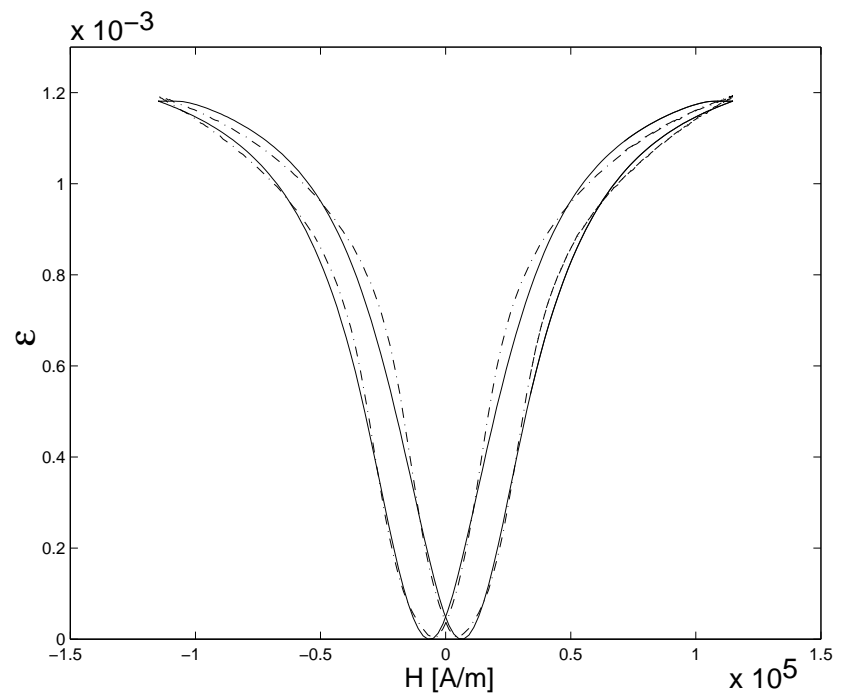

(b)

Figure 6: Comparison of model simulations (_- ) with experimental data $(-\cdot-)$, under a prestress of 1 ksi (6.9 MPa). (a) Magnetization of the magnetostrictive core, (b) total strain output by transducer.

\begin{tabular}{l|l|l|l|l}
\hline \multicolumn{1}{c|}{ Magnetization } & \multicolumn{1}{c|}{ Magnetostriction } & \multicolumn{1}{c|}{ Structural } & \multicolumn{1}{c}{ Load } & Geometry \\
\hline$a=6500 \mathrm{~A} / \mathrm{m}$ & $M_{0}=0.8 \times 10^{6} \mathrm{~A} / \mathrm{m}$ & $E=30 \times 10^{9} \mathrm{~Pa}$ & $k_{L}=2.5 \times 10^{6} \mathrm{~N} / \mathrm{m}$ & $L=0.115 \mathrm{~m}$ \\
$k=6000 \mathrm{~A} / \mathrm{m}$ & $\lambda_{s}=1000 \times 10^{-6}$ & $\rho=9250 \mathrm{~kg} / \mathrm{m}^{3}$ & $m_{L}=0.5 \mathrm{~kg}$ & $D=0.0127 \mathrm{~m}$ \\
$c=0.008$ & $\gamma_{1}=2.9 \times 10^{-15} \mathrm{~m}^{2} / \mathrm{A}^{2}$ & $c_{D}=1 \times 10^{6} \mathrm{Ns} / \mathrm{m}$ & $c_{L}=1 \times 10^{3} \mathrm{Ns} / \mathrm{m}$ & \\
$\alpha=0.03$ & $\gamma_{2}=-7.5 \times 10^{-28} \mathrm{~m}^{4} / \mathrm{A}^{4}$ & & & \\
$M_{s}=0.765 \times 10^{6} \mathrm{~A} / \mathrm{m}$ & & & & \\
$\xi=24.5 \times 10^{3} \mathrm{~Pa}$ & & & & \\
\hline
\end{tabular}

Table 1: Physical parameters and dimensions used in the coupled elastic-magnetic model.

\section{Concluding Remarks}

A coupled elastic-magnetic model for the magnetization and strain response of magnetostrictive transducers in response to applied currents has been presented. The model simulations accurately predict major loop behavior under mechanical preloads and quasi-static frequencies of operation. As indicated before [6], the model also accurately models the transducer's fundamental frequency within $5 \%$ of its measured value. Nevertheless, the model in its present format should be restricted to near-DC frequencies. Work is in progress to include AC losses and to extend the model to the frequency domain.

It is desirable to take advantage of the force simulations that the coupled model is able to generate. Work is in progress to gather experimental information for validation of the forces predicted by the model. Preliminary indications suggest that the involved stress amplitudes at input levels similar to those utilized in Section 5 are on the order of 3.2 MPa, or two orders of magnitude below the stress levels used in the experimental works of Pitman [4] and the theoretical developments of Jiles [5]. This fact explains why, for the operating regimes typical of the particular transducer unit tested, the coupled model did not provide a significant improvement over the uncoupled model presented previously [6].

We believe that the added accuracy and flexibility of the elastic-magnetic coupled model will enhance the control and performance capabilities of smart structure systems involving magnetostrictive materials, in particular those utilizing the full compression range of Terfenol-D, of about $700 \mathrm{MPa}$. The uncoupled formulation will still prove highly useful in applications where stress variations are less significant. 


\section{Acknowledgments}

The authors wish to thank David Jiles for his helpful suggestions regarding the modeling techniques employed here. Financial support for M.J.D. and A.B.F. was provided by the NSF Young Investigator Award \#CMS9457288 of the Division of Civil and Mechanical Systems. The work of R.C.S. was supported in part by the Air Force Office of Scientific Research under the grant AFOSR F49620-98-1-0180.

\section{References}

[1] W. F. Brown. "Irreversible magnetic effects of stress". Phys. Rev., 75(1):147-154, January 1949.

[2] R. M. Bozorth and H. J. Williams. "Effect of small stresses on magnetic properties". Rev. Mod. Phys., 17(1):7280, January 1945.

[3] R. R. Birss, C. A. Faunce, and E. D. Isaac. "Magnetomechanical effects in iron and iron-carbon alloys". J. Phys. D: Appl. Phys., 4:1040-1048, 1971.

[4] K. C. Pitman. "The influence of stress on ferromagnetic hysteresis". IEEE Trans. Magn., 26(5), Sept 1990.

[5] D. C. Jiles. "Theory of the magnetomechanical effect". J. Phys. D: Appl. Phys., 28:1537-46, 1995.

[6] M. J. Dapino, R. C. Smith, and A. B. Flatau. "An active and structural strain model for magnetostrictive transducers". In Proceedings of SPIE, Smart Structures and Materials 1998, volume 3329, pages 198-209, March 1998.

[7] M. J. Dapino, R. C. Smith, and A. B. Flatau. "A structural-magnetic strain model for magnetostrictive transducers". CRSC Technical Report CRSC-TR98-31 and IEEE Trans. Magn. Submitted 8/1998.

[8] D. C. Jiles and D. L. Atherton. "Theory of the magnetization process in ferromagnets and its application to the magnetomechanical effect". J. Phys. D: Appl. Phys., 17:1265-1281, 1984.

[9] D. C. Jiles and D. L. Atherton. "Theory of ferromagnetic hysteresis". J. Appl. Phys., 55(6):2115-2120, 1984.

[10] D. C. Jiles and D. L. Atherton. "Theory of ferromagnetic hysteresis". J. Magn. Magn. Mater., 61:48-60, 1986.

[11] B. D. Cullity. Introduction to magnetic materials. Addison-Wesley, 1972.

[12] S. Chikazumi. Physics of magnetism. R. E. Krieger, FL, 1984.

[13] F. T. Calkins. Design, analysis, and modeling of giant magnetostrictive transducers. PhD dissertation, Iowa State University, Ames, Iowa, 1997.

[14] D. C. Jiles, J. B. Thoelke, and M. K. Devine. "Numerical determination of hysteresis parameters for the modeling of magnetic properties using the theory of ferromagnetic hysteresis". IEEE Trans. Magn., 28(1):27-35, Jan 1992.

[15] E. W. Lee. "Magnetostriction and magnetomechanical effects". Reports on Prog. in Phys., 18:184-220, 1955.

[16] M. J. Sablik and D. C. Jiles. "A model for hysteresis in magnetostriction". J. Appl. Phys., 64(10):5402-5404, 1988.

[17] M. J. Dapino, F. T. Calkins, and A. B. Flatau. "Statistical analysis of Terfenol-D material properties". In Proceedings of SPIE, Smart Structures and Materials 1997, volume 3041, pages 256-267, March 1997.

[18] D. C. Jiles and D. L. Atherton. "Ferromagnetic hysteresis". IEEE Trans. Magn., MAG-19(5):2183-5, 1983.

[19] D. J. Craik and M. J. Wood. "Magnetization changes induced by stress in a constant applied field". J. Phys. D: Appl. Phys., 4:1009, 1971.

[20] F. T. Calkins, M. J. Dapino, and A. B. Flatau. "Effect of prestress on the dynamic performance of a Terfenol-D transducer". In Proceedings of SPIE, Smart Structures and Materials 1997, volume 3041, pages 293-304, 1997. 